\title{
Einleitende Worte zu Anwendungen des Zusammenwirkens
}

\section{Otto Richter}

Die möglichen Anwendungen des Zusammenwirkens künstlicher Intelligenz und lebender Entitäten sind nicht absehbar. Beinahe täglich wird in den Medien von Anwendungen berichtet, die gestern noch in den Bereich der Science-Fiction gehörten. Es gibt kaum eine Branche, die nicht von der digitalen Transformation betroffen ist. Diese Transformation hat ein enormes ökonomisches Potenzial, das in einer Studie der Fraunhofer-Allianz Big Data (2017) detailliert für einzelne Branchen analysiert wurde.

Aktuelle Entwicklungen aus dem Bereich Medizin und Gesundheitsversorgung betreffen intelligente Exoskelette für querschnittsgelähmte Menschen, intelligente persönliche Begleiter, Diagnose- und Therapie-Assistenten, Operationsroboter, empathische Assistenz im Wohnumfeld - um nur einige zu nennen. Der Beitrag von Marschollek und Wolf (in diesem Buch) zeigt als Beispiel den Impact der Wechselwirkung von künstlicher Intelligenz und körpernaher oder implantierter Systeme auf Medizin und die Gesundheitsversorgung.

Vielleicht unerwartet ist, dass die Digitalisierung in der Landwirtschaft schon sehr weit fortgeschritten ist. Laut einer von der Bitkom und dem Deutschen Bauernverband gemeinsam durchgeführten Studie aus dem Jahr 2016, in der 850 Betriebsleiter befragt wurden (Rohleder und Krüsken 2016), investieren $28 \%$ der befragten Betriebe gezielt in den Erwerb digitaler Kompetenzen. Typische Anwendungsbereiche sind die Viehhaltung, bei der beispielsweise Melkroboter, individuelle Fütterungssysteme und Gesundheitsüberwachung zum Einsatz kommen. Der Beitrag von Wolf zeigt neuere Trends umweltschonender

O. Richter $(\bowtie)$

Institut für Geoökologie der TU Braunschweig, Braunschweig, Deutschland

E-Mail: o.richter@tu-braunschweig.de

(C) The Author(s) 2021

R. Haux et al. (Hrsg.), Zusammenwirken von natürlicher und künstlicher

Intelligenz, https://doi.org/10.1007/978-3-658-30882-7_6 
Präzisionslandwirtschaft (Precision Agriculture) auf, die durch die Steuerung von Pflanzenbeständen mittels intelligenter Roboter realisiert wird, die beispielsweise Unkraut entfernen oder bedarfsgerecht Nährstoffe zuführen.

Im Focus des öffentlichen Interesses und der Medien steht das autonome Fahren, d. h. das Ersetzen der menschlichen Fähigkeiten im System Fahrer Fahrzeug - Umwelt durch Anwendung künstlicher Intelligenz. Dieses ist nur ein Aspekt möglicher Anwendungen der KI im Bereich der Mobilität. Umfassende Vision ist, Nutzer und deren Bedürfnisse, Verkehrsmittel und Infrastrukturen zu vernetzen, sodass Verkehrsflüsse und Mobilitätsverhalten in einem Gesamtsystem gesteuert werden können (Nationaler IT-Gipfel 2015). Der Beitrag von Jipp und Lemmer (im vorliegenden Buch) behandelt insbesondere den Aspekt der Wechselwirkung zwischen System und Nutzer aus menschlicher Sicht, der häufig weniger im Fokus steht.

\section{Literatur}

Fraunhofer-Allianz Big Data, Sankt Augustin. (2017, November). Potenzialanalyse „Künstliche Intelligenz ${ }^{\text {ec }}$ https://www.bigdata.fraunhofer.de/de/big-data/kuenstlicheintelligenz-und-maschinelles-lernen/potenzialanalyse--kuenstliche-intelligenz-.html.

Nationaler IT-Gipfel. (2015, November). Plattform Digitale Netze und Mobilität. https:// plattform-digitale-netze.de/publikationen/.

Rohleder, B., \& Krüsken, B. (2016, November 2). Bitkom und Deutscher Bauernverband zur Digitalisierung der Landwirtschaft. https://www.bitkom.org/sites/default/files/pdf/ Presse/Anhaenge-an-PIs/2016/November/Bitkom-Pressekonferenz-Digitalisierung-inder-Landwirtschaft-02-11-2016-Praesentation.pdf.

Open Access Dieses Kapitel wird unter der Creative Commons Namensnennung 4.0 International Lizenz (http://creativecommons.org/licenses/by/4.0/deed.de) veröffentlicht, welche die Nutzung, Vervielfältigung, Bearbeitung, Verbreitung und Wiedergabe in jeglichem Medium und Format erlaubt, sofern Sie den/die ursprünglichen Autor(en) und die Quelle ordnungsgemäß nennen, einen Link zur Creative Commons Lizenz beifügen und angeben, ob Änderungen vorgenommen wurden.

Die in diesem Kapitel enthaltenen Bilder und sonstiges Drittmaterial unterliegen ebenfalls der genannten Creative Commons Lizenz, sofern sich aus der Abbildungslegende nichts anderes ergibt. Sofern das betreffende Material nicht unter der genannten Creative Commons Lizenz steht und die betreffende Handlung nicht nach gesetzlichen Vorschriften erlaubt ist, ist für die oben aufgeführten Weiterverwendungen des Materials die Einwilligung des jeweiligen Rechteinhabers einzuholen.

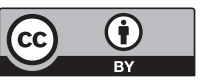

